$\mathbb{T}$ periodica polytechnica

Mechanical Engineering

$52 / 2(2008) 43448$

doi: 10.3311/pp.me.2008-2.01

web: http://www.pp.bme.hu/me

(c) Periodica Polytechnica 2008

RESEARCH ARTICLE

\section{Thermophysical properties of porous mineral-resin composites determined by a transient measurement technique}

\author{
Balázs Czél / Gyula Gróf
}

Received 2008-10-17

\begin{abstract}
Due to the continuous development of material sciences there is a constant demand to determine the thermophysical properties of new materials especially in the field of polymer technology. The temperature dependent volumetric heat capacity and thermal conductivity of two different porous mineral-resin composites were measured simultaneously by a transient technique developed by the authors. The technique includes one transient measurement and a genetic algorithm based evaluation method. We shortly present the principle of the technique and the measurement process and results for the two materials. The applied measurement technique can reduce the time consumption and the costs of the measurements compared to other well-known methods.
\end{abstract}

\section{Keywords}

inverse heat conduction · genetic algorithm · thermal conductivity $\cdot$ volumetric heat capacity $\cdot$ finite difference method

Balázs Czél

Department of Energy Engineering Engines, BME, H-1521 Budapest,, Hungary e-mail: czel@energia.bme.hu

\section{Gyula Gróf}

Department of Energy Engineering Engines, BME, H-1521 Budapest,, Hungary e-mail: grof@energia.bme.hu

\section{Introduction}

The volumetric heat capacity and the thermal conductivity are the most important material properties in the solution of engineering problems connected with the transient heat conduction. Due to the continuous development of material sciences, there is a steady demand for measuring the material properties of new materials especially in the field of polymer technology. The thermophysical properties of polymers are usually functions of the temperature. In this case using the familiar measurement methods one has to measure the properties at proper numbers of temperature levels which is usually a time consuming operation. A further challenge exists when finding both thermal conductivity and volumetric heat capacity, since two different experimental techniques are typically required.

The thermophysical properties of a material are usually determined by forcing the sample material into some kind of steady or non-steady heat conduction phenomenon. The temperature data are direct results of the measurement (with characteristic errors) and the temperature - space and/or temperature - time distributions are the solutions of the heat conduction problem. Determination of the properties requires determination of the unknown parameters appearing in the heat conduction equation. The task, defined such a way, is an inverse heat conduction problem (IHCP). There are a number of methods for solving such types of inverse problems [1,12], but none of them is generally accepted. We can find many different methods among the classical approaches: sequential method, modified Newton-Raphson method [8, 18, 19], Lagrange theory [6], simplex method [13], conjugate gradient method [2], etc.

The application of artificial intelligence based methods and evolutionary algorithms in IHCP has been spreading thanks to the rapid development of computer science and technology. There are two main methods: neural networks [7, 10, 14, 16] and genetic algorithms [3,5, 11, 15, 17]. In the literature sources mentioned above, the unknown parameter or unknown function sought for by the inverse problem is the boundary or the initial condition of a heat conduction problem, mostly in one dimension. In our case, the unknown parameters of the problem will be the volumetric heat capacity and the thermal conductivity and 
they appear as functions of the temperature.

The applied measurement technique offers simultaneous determination of the volumetric heat capacity and the thermal conductivity in case of linear temperature dependency and can reduce the time consumption and the costs of the measurement compared to other well-known methods. The advantages of this measurement technique can be maximized measuring polymers for the following reasons. The thermophysical properties of polymers generally have a temperature dependency. In the field of polymer technology, the demand for the measurements is high because of the huge variety of the materials. This way it is very important to perform the measurements fast and relatively low expense. Finally, the method works best with bad heat conductors. The measurement and evaluation method was modified and developed by the authors, based on the original idea of Kiss [9].

The aim of this article is to present the applied transient measurement technique and the measurement process and results for two different porous mineral-resin composites. These materials are often used for space filling in the PUR foaming resin tools. The knowledge of the thermophysical properties - especially the volumetric heat capacity and the heat conductivity are important for the proper thermal design. Moreover, the onsite measurement allows continuous and rapid improvement in production and quality.

\section{The measurement and evaluation method}

The measurement of the thermal conductivity is usually based on one-dimensional heat conduction and simple geometries like slabs, cylinders or spheres. In our measurement, a hollow cylindrical sample is used. The height of the cylinder is four times greater than the outer diameter to maintain the one-dimensional heat conduction assumptions. There is a core inserted in the hollow cylinder and an outer shield is fixed around the sample. The core and the shield have higher conductivity than the sample in order to maintain isothermal conditions on the outer and the inner surface of the sample. There are thermocouples inserted into the core and the shield (see Fig. 11.

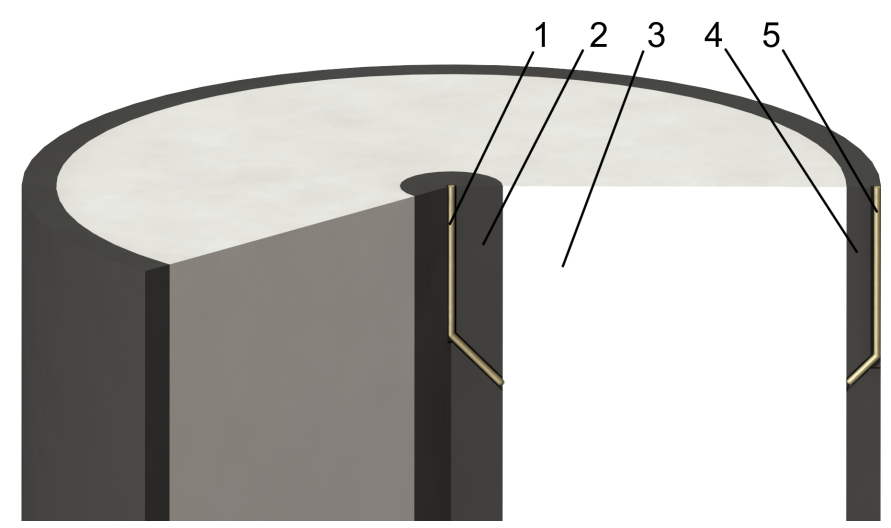

Fig. 1. Temperature measurement of the sample $(1-$ thermocouple in the core, 2 - core, 3 - sample, 4 - shield, 5 - thermocouple in the shield)

The measurement starts by heating up the sample to a pre- defined temperature. When the isothermal state is reached the heating process is stopped, the temperature registration and the forced air-cooling is started. During the cooling process the air temperature, the core temperature and the shield temperature are registered. The end of the cooling process - equalization of temperatures - is considered when the temperature of the core is close to the temperature of the airflow. During the evaluation procedure, the heat transfer coefficient should be known and it is determined by the help of a reference sample (see chapter 3 ). The evaluation is based on the core and shield temperature vs. time data.

The measurement equipment can be seen in Fig. 2. The cylindrical furnace can be moved in the vertical direction. During the heating, the furnace encircles the core-sample-shield system. When the isothermal state is reached, the furnace is moved down to its lower position. This way the forced air powered by the fan can cool the outer surface of the core-sample-shield system. The direction of the airflow is close to tangential at every point of the shield to ensure the homogenous distribution of the heat transfer coefficient. Four thermocouples are used to measure the temperature of the core, the shield, the furnace and the airflow. An electronic reference junction working at $50^{\circ} \mathrm{C}$ is used for the temperature measurement. The temperature data are registered via ICP Con data acquisition modules by a personal computer.

In this paper, we present the measurement results of two different samples. The samples and the reference sample can be seen in Fig. 3. Both samples are made of a granular material and epoxy resin. In case of Sample 1 the granular material is sand with $0.8-1.6 \mathrm{~mm}$ particle size, in case of Sample 2 the granular material is mullite (aluminium silicate) with $0-3 \mathrm{~mm}$ particle size. Both materials are porous with open pores. The reference sample which is used to determine the heat transfer coefficient is made of copper (see chapter 3 ).

The evaluation allows the determination of the volumetric heat capacity and the thermal conductivity as linear functions of the temperature using the temperature vs. time curves measured in the core and shield. The geometry and the initial and boundary conditions are known parameters, while the solution to the IHCP is found by a genetic algorithm based method developed by the authors [4]. To solve the inverse problem we have to solve the forward problem as well and this is achieved using a finite difference method. The genetic algorithm then uses the forward solution as an elementary step. The forward solution was optimized (time step, meshing) and verified in order to achieve the shortest CPU time and to keep the accuracy [4].

The genetic algorithms (GA) work similar to the biological evolution of nature. The GAs are mostly said to be optimization methods searching for the best solution of an ill-posed problem [5. 11]. The algorithm is working parallel on a number of candidate solutions known as entities. In this case one entity requires four parameters (equation 1), as we want to determine two linear functions. The linear functions are defined by two points, one point at the temperature of the airflow, the other one at the initial 


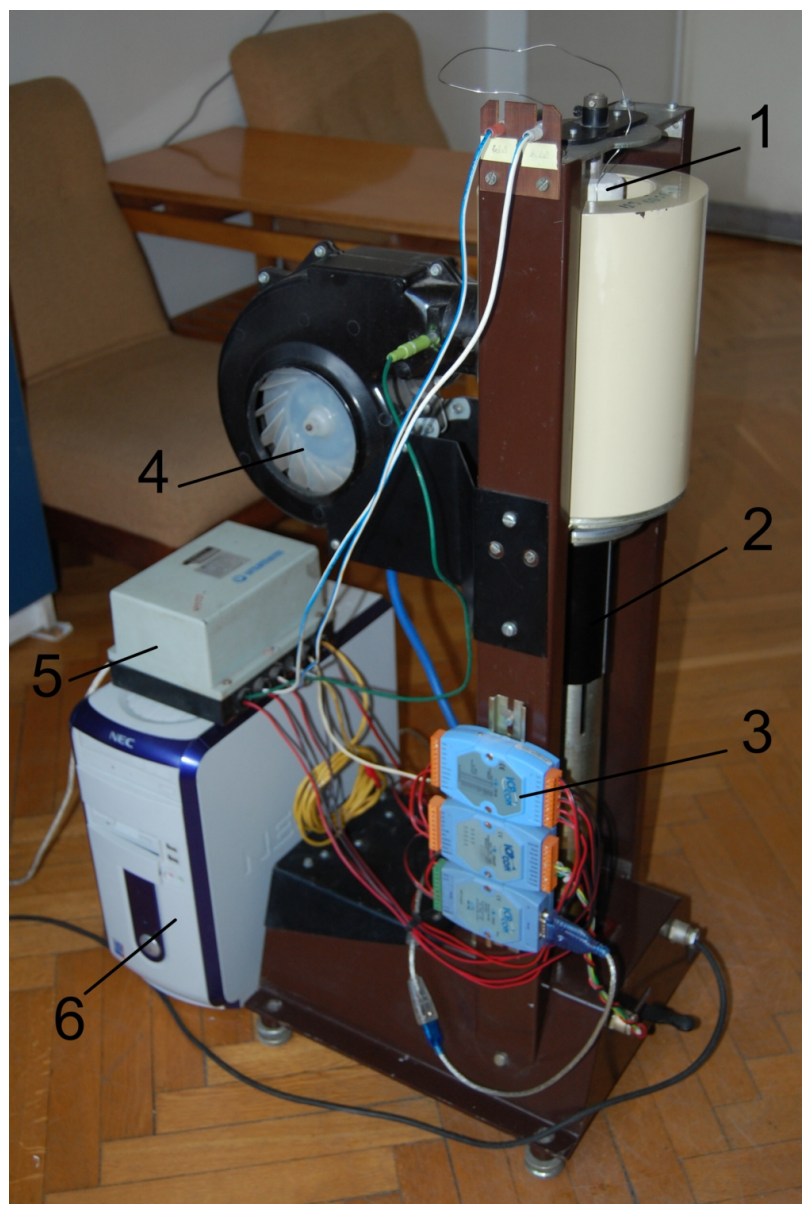

Fig. 2. The measuring equipment ( 1 - sample, 2 - furnace, 3 - data acquisition module, 4 - fan, 5 - electronic reference junction, 6 - personal computer)

temperature as this is the temperature range of the measurement.

$$
e=\left[\rho c\left(t_{o}\right), \rho c\left(t_{\infty}\right), k\left(t_{o}\right), k\left(t_{\infty}\right)\right]
$$

The search process is controlled by the objective function. In our case, the objective function is the minimization of the deviation between the measured and the calculated transient temperature data. The calculated temperature data refer to the temperature vs. time curves calculated by the forward solution for an entity created by the genetic algorithm. The objective function $(E)$ is calculated as the sum of the absolute deviations in 100 time steps for the inner surface and in 100 time steps for the outer surface of the sample as follows:

$$
E=\sum_{i=1}^{100}\left|t_{i, h 1}^{e}-t_{i, h 1}^{m}\right|+\sum_{i=1}^{100}\left|t_{i, h 2}^{e}-t_{i, h 2}^{m}\right|
$$

where $h 1$ indices refer to the inner surface, $h 2$ indices refer to the outer surface of the sample, $i$ indices refer to different time steps, $e$ indices refer to the temperature results of the actual entity and $m$ indices refer to the measurement data. The objective function is calculated for every entity. This way we can make a qualitative difference between the entities. The new entities are created using the best ones applying the genetic operators. The genetic operators contain many random operations. The algorithm stops when a preset number of entities are calculated or

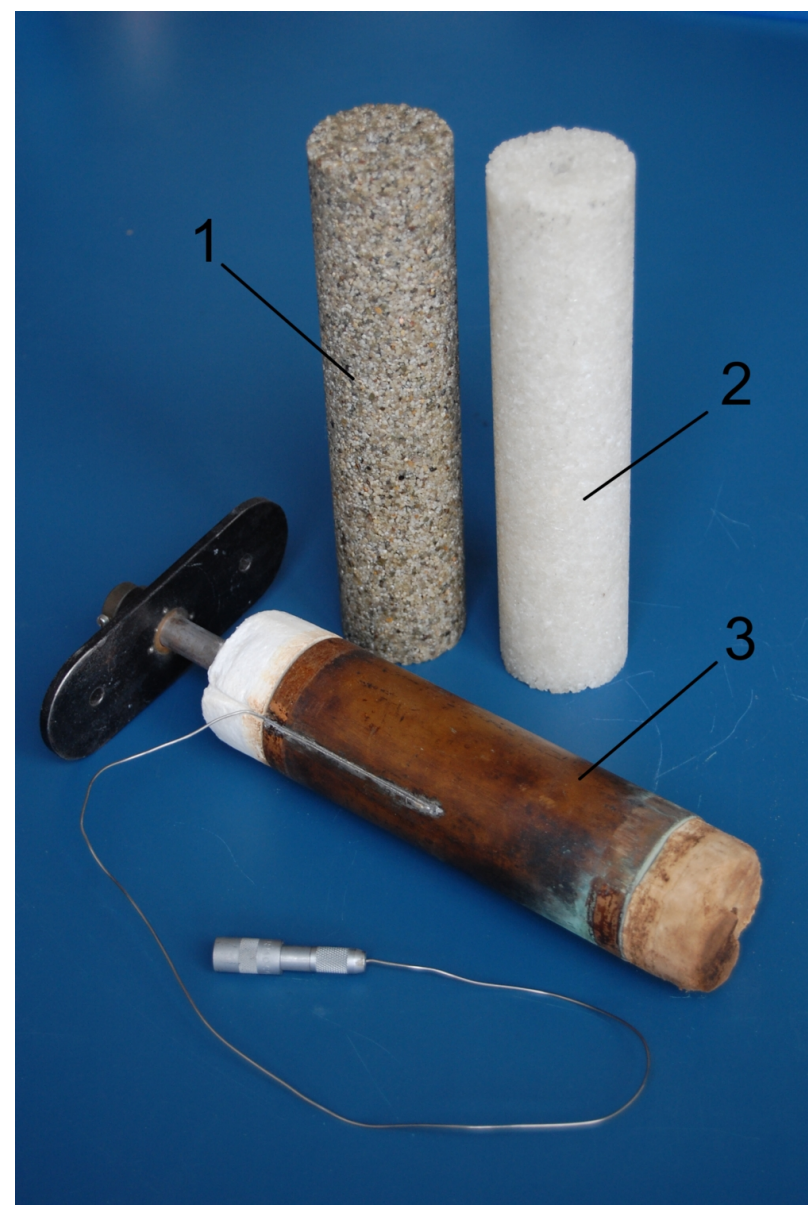

Fig. 3. Samples (1-Sample 1,2-Sample 2, 3 - reference sample)

the objective function reaches a preset limit. This means that every genetic run stops with a different objective function value and different combination of the material properties.

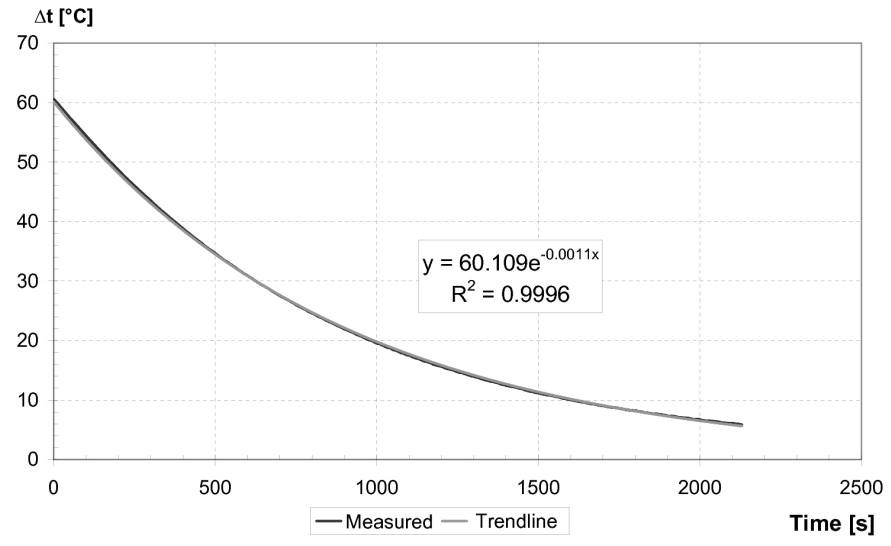

Fig. 4. Results of the reference sample measurement

\section{Determination of the heat transfer coefficient}

The heat transfer coefficient is determined using a reference sample (see Fig. 3). The reference sample is made of copper. The geometry of the reference sample is the same as the coresample-shield system. Efforts were taken to create a nearly homogenous airflow distribution at the surface of the reference sample. Because of this, a constant heat transfer coefficient is considered in time and space. In order to determine the heat 
transfer coefficient we perform a measurement process similar to the regular measurement process since only the temperature of the outer surface of the reference sample is recorded. As the copper has high thermal conductivity, the reference sample can be considered isothermal in space (lumped thermal capacity model) at every moment of the cooling process. In this case, the temperature vs. time curve of the cooling down can be described by the following equation:

$$
\Delta t(\tau)=t(\tau)-t_{\infty}=\left(t_{o}-t_{\infty}\right) \cdot e^{-\frac{h A}{m c} \tau}=\left(t_{o}-t_{\infty}\right) \cdot e^{-\frac{4 h}{d \rho c} \tau}
$$

where $t$ is the temperature, $\tau$ the time, $h$ is the heat transfer coefficient, $m$ is the mass, $c$ is the specific heat, $A$ is the area of the outer surface of the reference sample, $d$ is the outer diameter of the reference sample, $\rho$ is density. The indices $o$ and $\infty$ refer to the initial state and to the air flow respectively. The temperature results of the reference sample measurement can be seen in Fig. 4, where $\Delta t$ is the temperature difference between the reference sample and the airflow. An exponential trend line is fitted to the measured curve with a good accuracy in the following mathematical form:

$$
\Delta t(\tau)=t(\tau)-t_{\infty}=A \cdot e^{-B \tau}
$$

As we know the $A, B$ parameters of equation 4 from the curve fitting, the material properties and the geometry of the reference sample, we can calculate the heat transfer coefficient using equation 5.

$$
h=\frac{B d \rho c}{4}=47.6 \frac{\mathrm{W}}{\mathrm{m}^{2} \mathrm{~K}}
$$

This value of the heat transfer coefficient is a constant parameter of the measurement arrangement. If the geometry does not change, it is not necessary to measure it every time we test a new sample. It is sufficient to check it one or two times a year. If the temperature range of the measurement changes significantly it is recommended to check this value again.

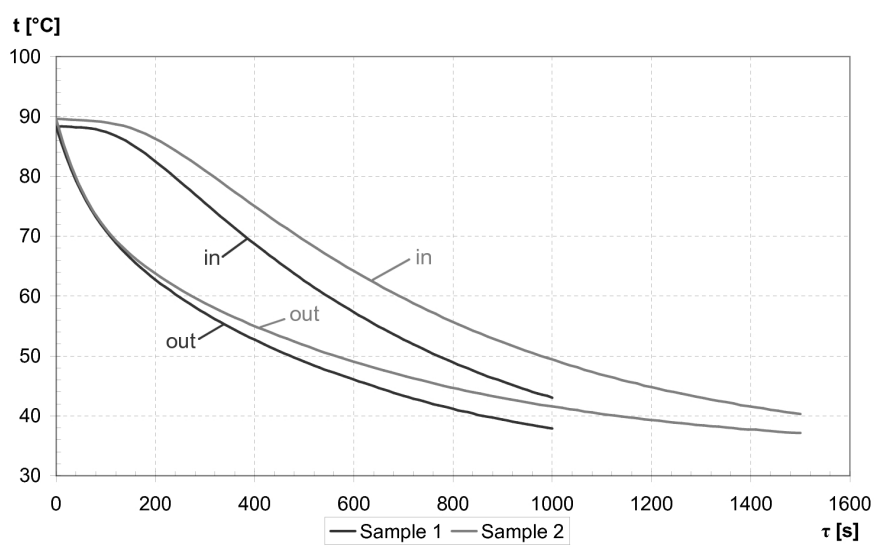

Fig. 5. Transient temperature results of the measurements
Tab. 1. Parameters of the evaluation

\begin{tabular}{llll}
\hline & Sample 1 & Sample 2 & \\
\hline$t_{0}$ & 88.2 & 89.5 & ${ }^{\circ} \mathrm{C}$ \\
$t_{\infty}$ & 30.7 & 33.2 & ${ }^{\circ} \mathrm{C}$ \\
$\tau_{\max }$ & 1000 & 1500 & $\mathrm{~s}$ \\
No. of genetic runs & 60 & 60 & - \\
Max. no. of generations & 100 & 100 & - \\
Searching limits of $\rho c$ & $1-4$ & $0.5-4$ & $\mathrm{MJ} /\left(\mathrm{m}^{3} \mathrm{~K}\right)$ \\
Searching limits of $k$ & $0.1-4$ & $0.1-4$ & $\mathrm{~W} /(\mathrm{mK})$ \\
\hline
\end{tabular}

\section{Results}

The measured transient temperature results of the two samples can be seen in Fig. 5 These two curves per sample are used in the evaluation. The parameters of the evaluation can be found in Table 1 . The evaluation is performed by a genetic algorithm based method. In order to get clear and reliable final results, we have to use the results of several genetic runs. The CPU time of one genetic run was about 20 minutes performed by a $2.33 \mathrm{GHz}$ Dual Core processor, so the CPU time of the evaluation procedure was about 20 hours for one measurement. The resulted material parameters of the genetic runs are plotted in function of the objective function $(E)$ in Fig. 6 and 7 . Every data point in the diagrams is a result of one genetic run. A clear convergence can be seen in all four diagrams. As the objective function decreases, the parameter in point tends to a single value like a comet. This value will be the final result of the evaluation. This final value can be estimated either by selecting the parameters with the lowest objective function value $(E)$ (best entity) or by calculating the average of the parameters below a limit of the objective function $(E)$. This limit of $E$ should be low enough to have a quasi-symmetrical region of the parameters below that limit. These two methods always give nearly the same final parameters.

Tab. 2. Final material properties of Sample 1 and 2

\begin{tabular}{llll}
\hline & Best entity & Average \\
\hline $\begin{array}{l}3 \text { Sample } 1 \\
\rho c \text { at } 30.7^{\circ} \mathrm{C}\end{array}$ & 1.240 & $\begin{array}{l}E<24^{\circ} \mathrm{C} \\
1.241\end{array}$ & $\mathrm{MJ} /\left(\mathrm{m}^{3} \mathrm{~K}\right)$ \\
\hline$\rho c$ at $88.2^{\circ} \mathrm{C}$ & 1.414 & 1.413 & $\mathrm{MJ} /\left(\mathrm{m}^{3} \mathrm{~K}\right)$ \\
\hline$k$ at $30.7^{\circ} \mathrm{C}$ & 0.676 & 0.679 & $\mathrm{~W} /(\mathrm{mK})$ \\
$k$ at $88.2^{\circ} \mathrm{C}$ & 0.676 & 0.673 & $\mathrm{~W} /(\mathrm{mK})$ \\
\hline Sample 2 & & $E<33.5^{\circ} \mathrm{C}$ & \\
\hline$\rho c$ at $33.2^{\circ} \mathrm{C}$ & 1.479 & 1.482 & $\mathrm{MJ} /\left(\mathrm{m}^{3} \mathrm{~K}\right)$ \\
$\rho c$ at $89.5^{\circ} \mathrm{C}$ & 1.510 & 1.505 & $\mathrm{MJ} /\left(\mathrm{m}^{3} \mathrm{~K}\right)$ \\
$k$ at $33.2^{\circ} \mathrm{C}$ & 0.598 & 0.598 & $\mathrm{~W} /(\mathrm{mK})$ \\
$k$ at $89.5^{\circ} \mathrm{C}$ & 0.509 & 0.506 & $\mathrm{~W} /(\mathrm{mK})$ \\
\hline
\end{tabular}

The final material properties using the best entity and the average methods can be found in Table 2 In the case of Sample 1 the volumetric heat capacity increases with the increasing temperature, while the thermal conductivity remains constant. In case of Sample 2 the volumetric heat capacity is nearly constant 

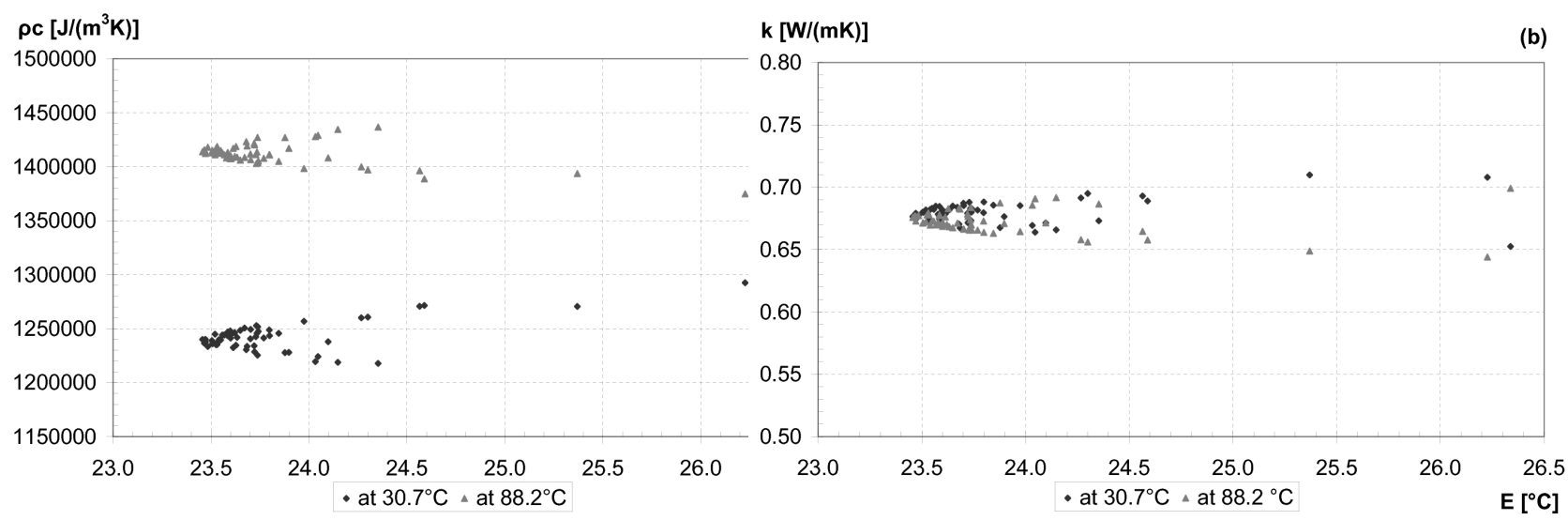

Fig. 6. Results of the evaluation for Sample 1
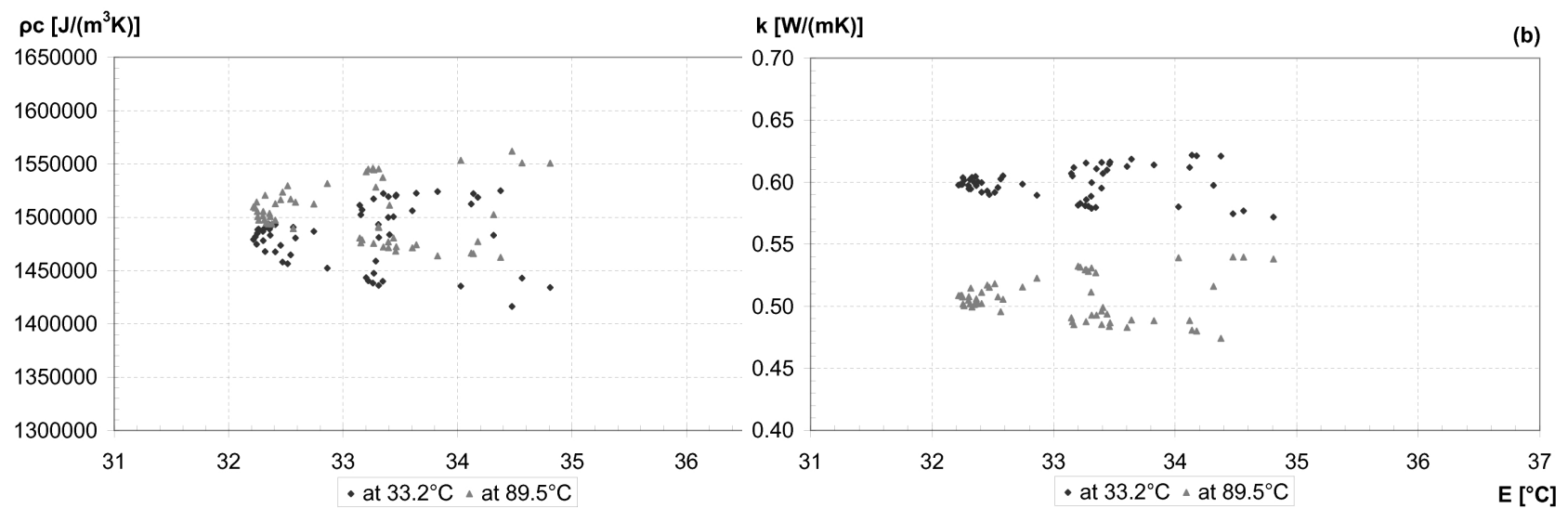

Fig. 7. Results of the evaluation for Sample 2

and the thermal conductivity decreases with the increasing temperature. The specific heat can be calculated easily considering constant density. The results can be found in Table 3 . The final material properties (using the best entity) plotted in function of the temperature are shown in Fig. 8.

Tab. 3. Specific heat of Sample 1 and 2

\begin{tabular}{llll}
\hline & $\begin{array}{l}\rho c \\
\text { (Best entity) }\end{array}$ & $\rho$ & $c$ \\
\hline & $\mathrm{J} /\left(\mathrm{m}^{3} \mathrm{~K}\right)$ & $\mathrm{kg} / \mathrm{m}^{3}$ & $\mathrm{~J} /(\mathrm{kgK})$ \\
\hline Sample 1 & & & \\
\hline at $30.7^{\circ} \mathrm{C}$ & 1240000 & 1652 & 751 \\
at $88.2^{\circ} \mathrm{C}$ & 1414000 & 1652 & 856 \\
\hline Sample 2 & & & \\
\hline at $33.2^{\circ} \mathrm{C}$ & 1479000 & 1968 & 752 \\
at $89.5^{\circ} \mathrm{C}$ & 1510000 & 1968 & 767 \\
\hline
\end{tabular}

In the genetic algorithm the linear material property functions are defined by two points. From the two data points the equation of the linear material property function can be calculated. These equations for the final material properties (best entity) together with the valid temperature ranges are as follows.

$$
\begin{aligned}
& c(t)_{\text {Sample 1 }}=1.83 \cdot t+695 \frac{\mathrm{J}}{\mathrm{kgK}} \\
& \lambda(t)_{\text {Sample } 1}=0.68 \frac{\mathrm{W}}{\mathrm{mK}} \quad(\text { constant }) \\
& 30.7^{\circ} \mathrm{C}<t<88.2^{\circ} \mathrm{C}<1+74 \frac{\mathrm{J}}{\mathrm{kgK}} \\
& c(t)_{\text {Sample 2 }}=0.27 \cdot t+7+0.65 \frac{\mathrm{W}}{\mathrm{mK}} \\
& \lambda(t)_{\text {Sample 2 }}=-0.0016 \cdot t+0.5^{\circ} \mathrm{C} \\
& 33.2^{\circ} \mathrm{C}<t<89
\end{aligned}
$$

\section{Conclusions}

The temperature dependent volumetric heat capacity and thermal conductivity of two different porous mineral-resin composites were determined simultaneously by a transient measurement technique developed by the authors. The measurement technique needs only one transient measurement. The material properties are calculated from the measured temperature data by a genetic algorithm based evaluation method. There are three main advantages of this method. The measurement takes only 1 or 2 hours. The volumetric heat capacity and the thermal conductivity can be determined simultaneously from the same transient temperature data. Finally, both material properties can be determined as linear functions of the temperature. The only disadvantage of the method is the CPU time of the evaluation. It 


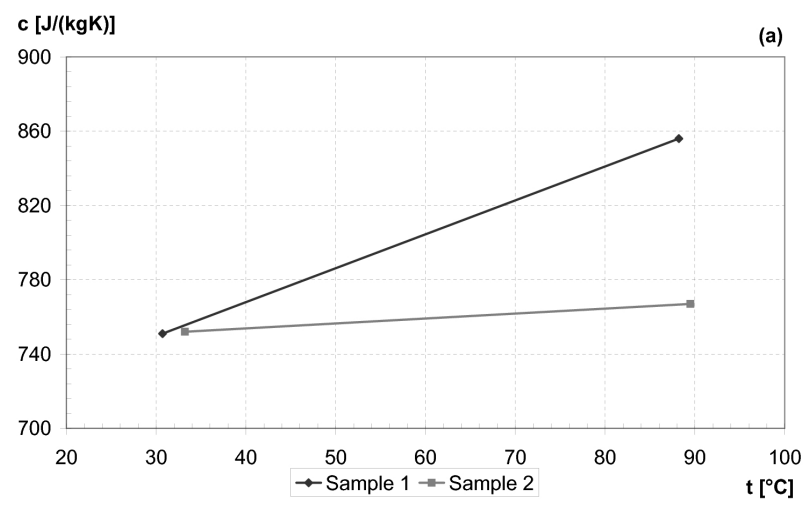

$\mathrm{k}[\mathrm{W} /(\mathrm{mK})]$

(b)

Fig. 8. Final material properties of Sample 1 and 2 (best entity)

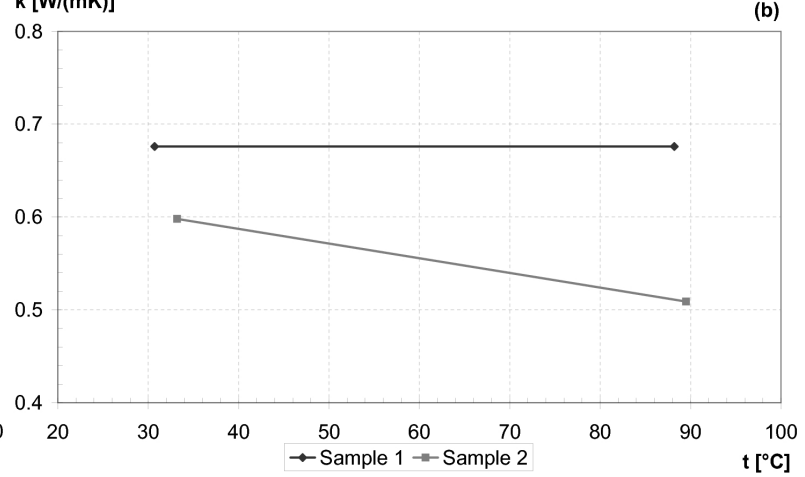

can take several hours, but due to the continuous development of computer technology the CPU time demand is less of a concern. Since the advantages of the presented measurement technique can be maximized measuring polymer materials, the method can be a very effective tool in polymer technology.

\section{References}

1 Beck J V, Blackwell B, Clair C. R. St, Inverse Heat Conduction: Ill-posed Problems, John Wiley, New York, 1985.

2 Chiwiacowsky L. D, Campos Velho H. F, Different Approaches for the Solution of a Backward Heat Conduction Problem, Inverse Problems in Engineering 11/6 (2003), 471-494, DOI 10.1080/1068276031000098027.

3 Chiwiacowsky L. D, Campos Velho H. F, Preto A. J, Stephany S, Identifying Initial Condition in Heat Conduction Transfer by a Genetic Algorithm: A Parallel Approach, Proc. XXIV Iberian Latin-american Congress on Computational Methods in Engineering (CILAMCE-2003), Ouro Preto (MG), Brazil, Unknown Month 29.

4 Czél B, Grof Gy, Inverse Heat Conduction Parameter Identification by Genetic Algorithm Based Method, ICHMT Digital Library 13/1 (2008), available at www. edata-center. com

5 Davis L (ed.), Handbook of Genetic Algorithms, Van Nostrand Reinhold, 1991.

6 Del Barrio E P, Multidimensional Inverse Heat Conduction Problems Solution via Lagrange Theory and Model Size Reduction Techniques, Inverse Problems In Science and Engineering 11/6 (2003), 515-539, DOI 10.1080/1068276031000114884.

7 Deng S, Hwang Y, Applying Neural Networks to the Solution of Forward and Inverse Heat Conduction Problems, International Journal of Heat and Mass Transfer 49 (2006), 4732-4750, DOI 10.1016/j.ijheatmasstransfer.2006.06.009.

8 Gróf Gy, The Thermal Diffusivity Measurement of Anthracite by the Flash Method in the Green and Calcined State, Periodica Polytechnica - Mechanical Engineering 46/2 (2002), 159-175.

9 Kiss L, Determination of Thermal Properties, 1983. CSc Thesis, HAS.

10 Krejsa J, Woodbury K A, Ratliff J D, Raudensky M, Assessment of Strategies and Potential for Neural Networks in the IHCP, Inverse Problems in Engineering 7/3 (1999), 197-213.

11 Mitchell M, An Introduction to Genetic Algorithms, A Bradford Book The MIT Press, Cambridge, Massachusetts, 1996.

12 Ozisik M. N, Orlande H. R B, Inverse Heat Transfer: Fundamentals and Applications, Taylor \& Francis, 2000.

13 Pohanka M, Woodbury K A, Woolley J, Obtaining Temperature Dependent Thermal Properties of Investment Casting Mold, Proc. IMECE 2002: International Mechanical Engineering Congress and Exposition, New Orleans LA, November 2002, DOI 10.1115/IMECE2002-32433, (to appear in print). 14 Raudensky M, Horsky J, Krejsa J, Usage of Neural Network for Coupled
Parameter and Function Specification Inverse Heat Conduction Problem, Int. Comm. Heat Mass Transfer 22/5 (1995), 661-670, DOI 10.1016/07351933(95)00052-Z.

15 Raudensky M, Woodbury K A, Kral J, Brezina T, Genetic Algorithm in Solution of Inverse Heat Conduction Problems, Numerical Heat Transfer, Part B: Fundamentals 28/3 (1995), 293-306, DOI 10.1080/10407799508928835.

16 Shiguemori E H, Harter F P, Campos Velho H F, da Silva J D S, Estimation of Boundary Conditions in Conduction Heat Transfer by Neural Networks, Tendencias em Mathematica Aplicada e Computacional 3/2 (2002), 189-195.

17 Woodbury K A, Application of Genetic Algorithms and Neural Networks to the Solution of Inverse Heat Conduction Problems: A tutorial, Inverse Problems in Engineering: Theory and Practice, Proceedings of the 4th International Conference on Inverse Problems in Engineering (Orlande H R B, ed.), Angra dos Reis, Brazil, May 2002, pp. 73-88.

18 Yang C, Estimation of Boundary Conditions in Nonlinear Inverse Heat Conduction Problems, Journal of Thermophysics and Heat Transfer 17/3 (2003), 389, DOI 10.2514/2.6780.

19 Zmywaczyk J, Numerical estimation of temperature-dependent thermophysical parameters by means of the inverse method-2D approach, Archives of thermodynamics 27/2 (2006), 1-18. 